\title{
IL-21 receptor signaling is integral to the development of Th2 effector responses in vivo
}

Anja Fröhlich, Benjamin J. Marsland, Ivo Sonderegger, Michael Kurrer, Martin R. Hodge, Nicola L. Harris and Manfred Kopf

Updated information and services can be found at:

http://bloodjournal.hematologylibrary.org/cgi/content/full/109/5/2023

Articles on similar topics may be found in the following Blood collections:

Immunobiology (4013 articles)

Signal Transduction (1930 articles)

Information about reproducing this article in parts or in its entirety may be found online at:

http://bloodjournal.hematologylibrary.org/misc/rights.dtl\#repub_requests

Information about ordering reprints may be found online at:

http://bloodjournal.hematologylibrary.org/misc/rights.dtl\#reprints

Information about subscriptions and ASH membership may be found online at:

http://bloodjournal.hematologylibrary.org/subscriptions/index.dtl

Blood (print ISSN 0006-4971, online ISSN 1528-0020), is published semimonthly by the American Society of Hematology, $1900 \mathrm{M} \mathrm{St}$, NW, Suite 200, Washington DC 20036.

Copyright 2007 by The American Society of Hematology; all rights reserved.

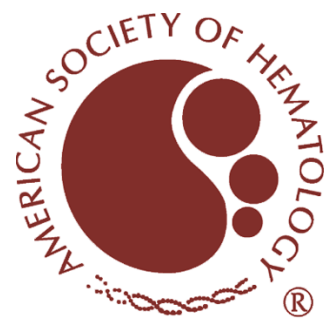




\title{
IL-21 receptor signaling is integral to the development of Th2 effector responses in vivo
}

\author{
Anja Fröhlich, ${ }^{1}$ Benjamin J. Marsland, ${ }^{1}$ Ivo Sonderegger, ${ }^{1}$ Michael Kurrer, ${ }^{2}$ Martin R. Hodge, ${ }^{3}$ \\ Nicola L. Harris, ${ }^{4}$ and Manfred Kopf ${ }^{1}$ \\ ${ }^{1}$ Institute of Integrative Biology, Molecular Biomedicine, Swiss Federal Institute of Technology (ETH) Zürich, Switzerland; ${ }^{2}$ Deparment of Pathology, University of \\ Zürich, Switzerland; ${ }^{3}$ Inflammation Division, Millennium Pharmaceuticals, Cambridge, MA; ${ }^{4}$ Institute of Integrative Biology, Environmental Biomedicine, ETH \\ Zürich, Switzerland
}

Interleukin 21 (IL-21) is a member of the common $\gamma$-chain family of cytokines, which influence a broad spectrum of immunologic responses. A number of studies have examined the function of IL-21, but its specific role in Th1/Th2-cell differentiation and related effector responses remains to be clarified. Thus, we generated IL-21R-deficient mice and have investigated the role of IL-21R signaling using a series of in vivo experimentally induced disease models. We first ad- dressed the role of IL-21R signaling in Th2 immune responses by examining allergic airway inflammation, and NippostrongyIus brasiliensis and Heligmosomoides polygyrus antihelminth responses. In each of these systems, IL-21R signaling played a clear role in the development of Th2 responses. Comparatively, IL-21R signaling was not required for the containment of Leishmania major infection or the development of experimental autoimmune myocarditis, indicative of competent Th1 and Th17 responses, respectively. Adoptive transfer of $T$ cells and analysis of IL-21 $R^{+/+} / / L-21 R^{-/-}$ chimera mice revealed that IL-21R-signaling was central to Th2-cell survival or migration to peripheral tissues. Overall, our data show IL-21 plays a crucial role in supporting polarized Th2 responses in vivo, while appearing superfluous for Th1 and Th17 responses. (Blood. 2007;109:2023-2031)

(c) 2007 by The American Society of Hematology

\section{Introduction}

Cytokines are key players in shaping immune responses against both self and foreign antigens, influencing the induction of either tolerance or effective immunity. While cytokines have a broad spectrum of activities, distinct levels of redundancy due to the sharing of common receptors, or receptor subunits, help ensure that appropriate immune responses develop. Interleukin-2 (IL-2), IL-4, IL-7, IL-9, and IL-15 belong to the type I cytokine family, and their receptors share the common $\gamma_{c}$ chain and signal via the Jak/STAT pathway. ${ }^{1-5}$ These cytokines play crucial roles in T-cell responses including cell proliferation, differentiation, and maintaining memory populations. IL-21 is the most recently described member of this cytokine family, ${ }^{6,7}$ and a series of studies have shown that it has pleiotropic effects upon the development of immune responses. The main source of IL-21 is activated $\mathrm{CD}^{+}{ }^{+} \mathrm{T}$ cells, however numerous other cell types express the receptor. ${ }^{6}$ It has been shown to promote expansion of natural killer (NK) cells and augment their antitumor activity ${ }^{8}$ but also to inhibit dendritic cell (DC) activation and maturation in vitro. ${ }^{9}$ Furthermore, IgE isotype switching is dysregulated in the absence of IL-21 receptor signaling, indicative of a regulatory role for IL-21. ${ }^{10}$ The data to date concerning the role of IL-21 in Th1 and Th2 responses are controversial. ${ }^{11}$ Addition of IL-21 to in vitro T-cell cultures has been shown to have no influence on the production of IL-4 and IL-5, while in vivo, exogenous IL-21 can reduce the infiltration of eosinophils into the airways (generally considered to be IL-5 dependent). ${ }^{12} \mathrm{~A}$ further study has shown that IL-21 mRNA was exclusively detected in in vitro and in vivo polarized Th2 cells, but not Th1 cells. ${ }^{13}$ This study also showed that IL-21 inhibited IFN- $\gamma$ production by Th1 cells in vitro, although neither TNF- $\alpha$ production nor T-bet expression was compromised..$^{13}$ In contrast, Strengell et $\mathrm{al}^{14}$ showed that IL-21 could induce expression of genes involved in Th1 responses including IFN- $\gamma$, the IL-12R $\beta$ chain, and T-bet. In an attempt to clarify the role of IL-21 in both Th1 and Th2 immune responses, we have generated mice deficient in the IL-21 receptor, and extensively assessed their ability to mount Th1 and Th2 responses in vivo. We found IL-21R expression was central to the development of Th2-driven allergic airway inflammation as shown by impaired Th2 cytokine production, infiltration of eosinophils, and airway hyperresponsiveness. In support of prior publications, antibody isotype switching was also dysregulated, characterized by reduced IgG1 and $\operatorname{IgA}$ and increased $\operatorname{IgE}$ production. In line with these data, Th2 responses against the parasites Nippostrongylus brasiliensis and Heligmosomoides polygyrus were similarly impaired. However, this immune dysregulation was not a general phenomenon, as the development of Th1 immune responses against Leishmania major and Th17-driven experimental autoimmune myocarditis (EAM) were normal. Overall, our data from in vivo disease models support a central role for IL-21 signaling in Th2, but not Th1 or Th17, immune responses.

\section{Materials and methods}

\section{Mice and pathogens}

C57BL/6 and BALB/c wild-type mice were obtained from Charles River (Sulzfeld, Germany). IL-21R-deficient mice were generated as described in "Generation of IL-21R-deficient mice" and backcrossed more then 5 generations onto the C57BL/6 background and 4 generations onto the
Submitted May 9, 2006; accepted October 10, 2006. Prepublished online as Blood First Edition Paper, October 31, 2006; DOI 10.1182/blood-2006-05-021600.

The publication costs of this article were defrayed in part by page charge payment. Therefore, and solely to indicate this fact, this article is hereby marked "advertisement" in accordance with 18 USC section 1734.

C 2007 by The American Society of Hematology 
BALB/c background. Mice were maintained in specific pathogen-free conditions at the BioSupport (Zürich, Switzerland) animal facility in isolated ventilated cages. Animals used in experiments were between 8 and 10 weeks of age. $N$ brasiliensis was maintained by passage through Lewis rats; $H$ polygyrus was passaged through $\mathrm{IL}-4^{-1-}$ mice on a C57BL/6 background. Mice were infected subcutaneously with 550 live $N$ brasiliensis L3 worms or orally with 200 L3 H polygyrus worms and killed at day 14 after infection. For infection with $L$ major, stationary-phase promastigotes were collected from in vitro culture in biphasic Novy-Nicolle-McNeal (NNN) blood agar medium, and 1 to $2 \times 10^{6}$ parasites were injected subcutaneously into the right hind footpad. The infection was monitored by measuring footpad swelling with a metric caliper (Kroepelin, Schluchtern, Germany). Mice were killed and tissues taken at the indicated time points. All animal experimental procedures were approved by the Zürich animal committee.

\section{Genotyping of IL-21R-deficient mice}

DNA was isolated from tails of mice by tissue lysis and DNA precipitation. For the polymerase chain reaction (PCR), the following primers were used: IL-21R-forward, 5'-GCT AGC ACT TCT ATA GGC AAA GGG-3'; IL-21R-reverse, 5'-CAG CTG AGC TTT GTG GGA GG-3'; and NEO 3193, 5'-CGA GAC TAG TGA GAC GTG CTA CTT CC-3'. Using Platinum Taq (Invitrogen, Frederick, MD), the reaction was performed in a T 3000 Thermocycler (Biometra, Goettingen, Germany) over 35 cycles with an annealing temperature of $58^{\circ} \mathrm{C}$.

\section{OVA-induced airway inflammation}

Mice were immunized by intraperitoneal injection with $100 \mu \mathrm{g}$ OVA (grade V; Sigma-Aldrich, Buchs, Switzerland) in $200 \mu \mathrm{L}$ alum adjuvant (SERVA; Electrophoresis, Heidelberg, Germany). Ten days later, mice were challenged on the 4 following days by intranasal inoculation with $100 \mu \mathrm{g}$ OVA in $50 \mu \mathrm{L}$ PBS.

\section{Measurement of airway responsiveness}

One day after last intranasal challenge with OVA, mice were placed in individual unrestrained whole-body plethysmograph chambers (Buxco Electronics, Petersfield, United Kingdom). Airway responsiveness was assessed in mice by inducing airflow obstruction with aerosolized methacholine-chloride (MetCh). This procedure estimates total pulmonary airflow in the upper and lower respiratory tracts. The chamber pressure was used as a measure of the difference between thoracic expansion (or contraction) and air volume removed from (or added to) the chamber during inspiration (or expiration). Pulmonary airflow obstruction was assessed by measuring PenH using BioSystem XA software (Buxco Electronics). Measurements of MetCh responsiveness were obtained by exposing mice for 3 minutes to incremental doses of aerosolized MetCh (Aldrich Chemie, Steinheim, Germany) and monitoring the breathing pattern for 5 minutes after initiation of aerosol dose.

\section{Collection and analysis of BAL cells}

Two days after the last intranasal challenge with OVA, mice were killed by $\mathrm{CO}_{2}$ inhalation. The trachea was cannulated and bronchoalveolar lavage (BAL) was performed by flushing the airways 3 times with $0.4 \mathrm{~mL}$ PBS. Total BAL cells were counted using a Coulter Counter (IG Instruments, Zürich, Switzerland) and spun onto glass slides using a Cytospin 2 (Shandon Southern Products, Astmoor, England). Following fixation with methanol for 2.5 minutes, cells were stained with undiluted May-Grünwald solution (Fluka, Buchs, Switzerland) for 3 minutes. A further staining was performed in a 50\% May-Grünwald solution for 3 minutes. In a last step, cells were stained in 7\% Giemsa solution (Fluka) for 12 minutes. Slides were rinsed with water and air-dried overnight. Dried cells were embedded in Eukit solution under glass coverslips. Percentages of macrophages, lymphocytes, neutrophils, and eosinophils were determined microscopically by counting 200 cells/sample using standard morphologic and cytochemical criteria. Total numbers of eosinophils were derived by multiplying the total number of leukocytes by the percentage of eosinophils identified morphologically.

\section{Generation of bone marrow chimeras}

Recipient mice were lethally irradiated ( $9.5 \mathrm{~Gy}$ ) by using a 60 Cobalt source and were injected intravenously with $1 \times 10^{7}$ Thy 1 -depleted bone marrow (BM) cells that were collected from tibias and femurs of donor mice. After 6 weeks, reconstitution of the hematopoietic system was assessed by fluorescence-activated cell sorting (FACS) analysis.

\section{Adoptive transfer of SMARTA2 (Sm2) transgenic T cells}

Naive CD4 T cells were isolated from spleens of Sm2 or IL-21R ${ }^{-1-} \mathrm{Sm} 2$ mice and $1 \times 10^{7} \mathrm{~T}$ cells were adoptively transferred to C57BL/6. One day after transfer, mice were immunized intraperitoneally with $50 \mu \mathrm{g} \mathrm{GP}_{61-80}$ peptide in alum adjuvant. Ten days after immunization, mice were challenged intranasally with $25 \mu \mathrm{g} \mathrm{GP} 61-80$ on 2 consecutive days. Five days after the first challenge, mice were killed and cells isolated for analysis.

\section{TCR transgenic T-cell-DC coculture}

Naive $\mathrm{CD} 4^{+} \mathrm{T}$ cells were isolated from spleens by magnetic-activated cell sorting (MACS) bead separation following the manufacturer's instructions (Miltenyi Biotech, Bergisch Gladbach, Germany) and were found to be more than $90 \% \mathrm{CD}^{+} \mathrm{CD} 2 \mathrm{~L}^{\text {high }}$ by subsequent FACS analysis. DCs were isolated from spleens of naive wild-type or IL-21R-deficient mice as described previously. ${ }^{15}$ Isolated T cells $\left(6.5 \times 10^{4}\right.$ cells/well $)$ and DCs $\left(1.4 \times 10^{4}\right.$ cells/well $)$ were cultured in 96 -well plates in the presence of $\mathrm{GP}_{61-80}$ peptide at a concentration of $10 \mathrm{nM}$. After 24 hours, T cells were analyzed for expression of activation markers: cells were incubated with a biotinylated anti-CD40L antibody in IMDM at $37^{\circ} \mathrm{C}$. After 4 hours, cells were washed twice with PBS/0.1\% BSA and incubated with PE-labeled anti-CD62L, APC-labeled anti-CD25, and PerCp-labeled Streptavidin (BD Pharmingen, San Diego, CA) for 25 minutes at $4^{\circ} \mathrm{C}$. Cells were washed extensively and analyzed by flow cytometry. To assess proliferation, purified SM2 transgenic T cells were labeled with $5 \mu$ M CFSE (Molecular Probes, Leiden, The Netherlands) by incubation at room temperature for 7 minutes. After extensive washing with IMDM, CFSE-labeled T cells were cultured in the presence of $\mathrm{GP}_{61-80}$ and DCs. After 3 days, T cells were harvested and stained with PerCp-labeled anti-CD4 antibody (BD Pharmingen), and the $\mathrm{CD} 4^{+} \mathrm{T}$ cells were analyzed for CFSE staining by flow cytometry.

\section{ELISA measurement of antibodies and cytokines}

At the indicated time points, BAL fluid, serum, or culture supernatant was analyzed for either total IgE, specific IgG1, specific IgA, IL-4, or IFN- $\gamma$. Ninety-six-well plates (Maxisorp; Nunc, Roskilde, Denmark) were coated with anti-IgE, anti-IFN- $\gamma$, or anti-IL-4 at $5 \mu \mathrm{g} / \mathrm{mL}$ in $50 \mu \mathrm{L}$ PBS overnight at $4^{\circ} \mathrm{C}$. For antigen-specific enzyme-linked immunosorbent assays (ELISAs), plates were coated with OVA at $50 \mathrm{mg} / \mathrm{mL}$ overnight at $4^{\circ} \mathrm{C}$. Between all of the following steps, plates were washed 5 times with PBS. Coated plates were blocked with PBS/1\% BSA for 2 hours at room temperature. Samples from individual mice/cultures were serially diluted in PBS/0.1\% BSA as indicated, followed by incubation at room temperature for 2 hours. Thereafter, alkaline phosphatase-labeled goat antimouse antibodies to IgE, IgG1, IgA (Southern Biotechnology Associates, Birmingham, AL), IL-4, or IFN- $\gamma$ were added at room temperature for 2 hours, followed by addition of the substrate p-nitrophenyl phosphate (Sigma-Aldrich). Optical density (OD) was determined at $405 \mathrm{~nm}$.

\section{Proliferation assay}

A single-cell suspension was made from the indicated lymph nodes by gentle teasing through 70- $\mu$ m nylon cell strainers (Falcon; Becton Dickinson, Heidelberg, Germany) and cells were washed through the strainers with IMDM into 50-mL (Falcon) tubes. Antigen at the indicated concentrations was diluted in 96-well plates and $5 \times 10^{5}$ cells were added per well. Cells were cultured for 72 hours at $37^{\circ} \mathrm{C}$ with $\left[{ }^{3} \mathrm{H}\right]$ thymidine added $(1 \mu \mathrm{Ci}$ 
$[0.037 \mathrm{MBq}] /$ well) for the last 12 hours. Total $\left[{ }^{3} \mathrm{H}\right]$ thymidine incorporation was measured as an indicator of cell proliferation.

\section{Intracellular cytokine staining and FACS analysis}

Approximately $5 \times 10^{5}$ cells from either BAL samples or in vitro culture were stimulated with PMA $\left(10^{-7} \mathrm{M}\right)$ and ionomycin $(1 \mu \mathrm{g} / \mathrm{mL})$. For the final 2 hours, brefeldin A $(10 \mu \mathrm{g} / \mathrm{mL})$ was added to the cultures. Thereafter, cells were washed with $\mathrm{PBS} / 0.1 \%$ BSA and incubated with anti-CD32/ $\mathrm{CD} 16 \mathrm{mAb}$ for 30 minutes at $4^{\circ} \mathrm{C}$ to block $\mathrm{Fc}$ binding. After another washing step, cells were stained with PerCp-labeled anti-CD4 mAb (BD PharMingen) for 15 minutes at $4^{\circ} \mathrm{C}$. For basophil detection, blood samples were stained with FITC-labeled IgE and with PE-labeled CD49b after blocking. Subsequently, cells were washed with PBS/0.1\% BSA, then again in PBS, and fixed with $2 \%$ paraformaldehyde for 20 minutes at room temperature. For intracellular staining, fixed cells were then incubated in permeabilization buffer (0.5\% saponin/PBS/1\% BSA) containing APClabeled anti-IFN- $\gamma$ and PE-labeled anti-IL-4 mAb (BD PharMingen) for 30 minutes at room temperature. Cells were washed twice in permeabilization buffer and then resuspended in PBS/1\% BSA and analyzed by flow cytometry (FACSCalibur; Becton Dickinson) and Flowjo software (Tree Star, Ashland, OR).

\section{Induction of experimental autoimmune myocarditis and histologic assessment}

A murine heart muscle-specific peptide derived from $\alpha$-myosin $\mathrm{H}$ chain (Ac-RSLKLMATLFSTYASADR-OH) was used as antigen. The peptide (purity, 85\%; ANAWA Biochemical Services and Products, Zürich, Switzerland) was dissolved in CFA (Difco, Detroit, MI) and emulsified 1:1 with PBS. Mice were immunized subcutaneously with $100 \mu \mathrm{g} / 0.2 \mathrm{~mL}$ on days 0 and 7. Sham-immunized controls were injected with CFA emulsified with PBS alone. Fourteen days after the second immunization, mice were killed, and their hearts removed, fixed in $4 \%$ neutral buffered formalin, and processed for hematoxylin and eosin staining. The glass slides were coded and evaluated by a pathologist. For diagnosis of myocarditis, an inflammatory infiltrate forming foci between muscle fibers or surrounding individual myocytes, with or without associated myocyte necrosis or apoptosis, was considered essential. A vague increase in interstitial cellularity was not considered sufficient for diagnosis. Myocarditis was scored on a semiquantitative scale using grades from 0 to 4 ( 0 indicates no inflammatory infiltrates; 1, small foci of inflammatory cells between myocytes or inflammatory cells surrounding individual myocytes; 2, larger foci of 100 inflammatory cells or involving 30 myocytes; $3,10 \%$ of a myocardial cross-section involved; and 4, 30\% of a myocardial cross-section involved).

\section{Microscopy}

Histology samples and blood smears were viewed using an Axioplan 2 microscope (Carl Zeiss, Jena, Germany) equipped with a Zeiss PlanApochromat $63 \times / 1.4$ numerical aperture (NA) oil or a Zeiss Plan-Neofluor $40 \times / 0.75$ NA HF objective and a Retiga Exi CCD camera (Qimaging, Burnaby, BC, Canada) with Openlab imaging software (Improvision, Coventry, United Kingdom). Adobe Photoshop (Adobe Systems, San Jose, CA) was used for image processing.

\section{Statistics}

Statistical significance was analyzed by the Student $t$ test. Unless otherwise indicated, data represent mean \pm standard deviation, with $P$ values less than .05 statistically significant.

\section{Results}

\section{Generation of IL-21R-deficient mice}

IL-21 $\mathrm{R}^{-1-}$ mice were generated by homologous recombination. The targeting vector for IL-21R contained regions of exons 2 and 5 and was designed to replace the intervening IL-21R coding sequence with a 3.3-kb neomycin resistance cassette (Figure 1A). The target vector was electroporated into $129 \mathrm{~Sv}$ ES cells, and recombinant ES clones were identified by Southern blot analysis of genomic DNA and microinjected into C57BL/6 blastocysts. Germline transmission of the targeted allele was confirmed by Southern blot of tail genomic DNA. The resulting chimera mice were backcrossed onto either C57BL/6 or BALB/c strains. Mice were genotyped by PCR as described in "Materials and methods" (Figure 1B). The homozygous IL-21R-deficient mice were viable and fertile and showed no gross developmental abnormalities. The absence of IL-21R mRNA in cells derived from knock-out mice was confirmed by reverse-transcription (RT)-PCR (Figure 1C). Proportions of monocyte, lymphocyte, and granulocyte populations appeared normal in the IL-21 $\mathrm{R}^{-1-}$ mice; however, circulating IgE levels were increased approximately 2 -fold in the IL-21R $\mathrm{R}^{-1-}$ mice (data not shown).

\section{IL-21R signaling is required for Th2 responses against helminth parasites}

Given the controversy over the role of IL-21 in Th2 immune responses, we chose to investigate 2 well-defined in vivo models of Th2 immunity, specifically, infection with the helminth parasites Nippostrongylus brasiliensis $(\mathrm{Nb})$ and Heligmosomoides polygyrus (Hp). C57BL/6 and IL-21R $\mathrm{R}^{-1-}$ mice on a C57BL/6 background were infected subcutaneously with $\mathrm{Nb}$ and 14 days later the infiltration of cells into the airways was assessed. The infiltration of both eosinophils and lymphocytes was reduced in the absence of IL-21R signaling (Figure 2A), supporting a role for IL-21 in the development of Th2 cells and their effector function. In addition, $\mathrm{IgA}$ and $\mathrm{IgG} 1$ levels in BAL fluid and serum were decreased in IL-21R $\mathrm{R}^{-1-}$ mice, while IgE was similar (Figure $2 \mathrm{~B}$ and data not shown). We next examined the immune response against the intestinal helminth, Hp. C57BL/6 and $\mathrm{IL}-21 \mathrm{R}^{-1-}$ mice on a C57BL/6 background were infected via oral gavage with $\mathrm{Hp}$. On day 14 after infection, the proportion of basophils both in the blood and the spleen was significantly reduced in the absence of IL-21R signaling (Figure 3A). Similarly, the proportion of eosinophils

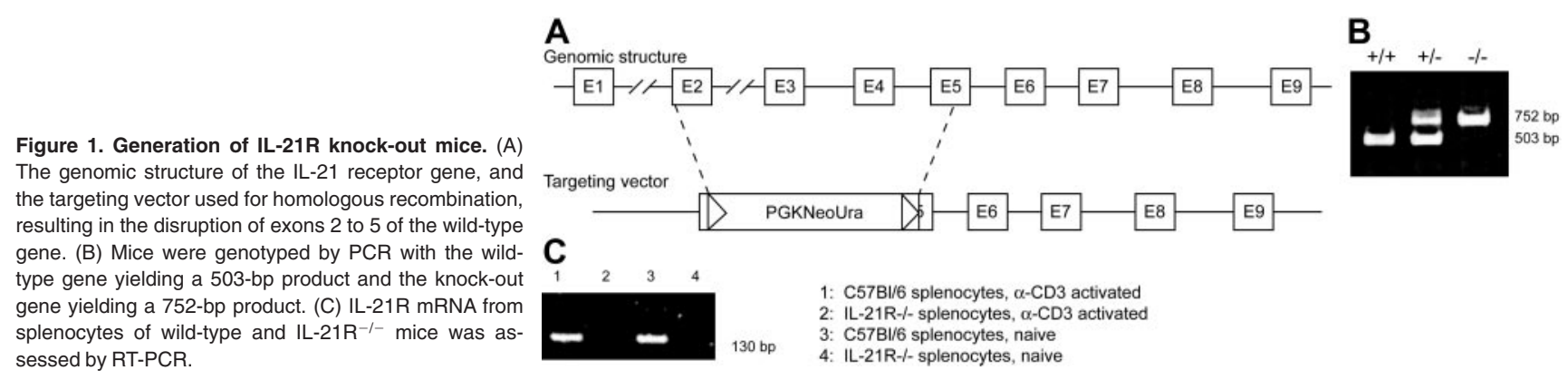


A

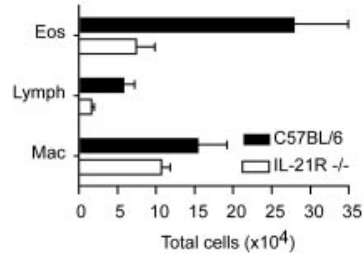

B

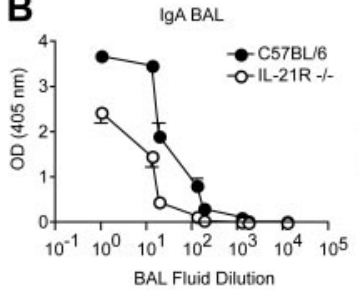

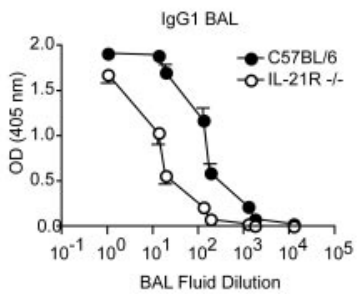

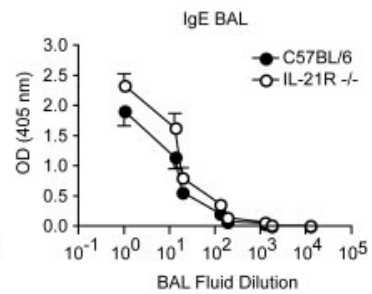

Figure 2. IL-21R signaling is required for Th2 responses against Nippostrongylus brasiliensis. C57BL/6 and IL-21R-deficient mice were infected subcutaneously with $\mathrm{Nb}$. On day 14 after infection, BAL was performed and (A) the total number of eosinophils, lymphocytes, and macrophages was determined by total and differential cell counts. (B) Total IgA, IgG1, and IgE in the BAL fluid were assessed by ELISA. Data show averages \pm SD of a representative experiment using 5 to 6 mice per group. Similar results were obtained in an independent experiment.

circulating in the blood was also reduced in the IL-21 $\mathrm{R}^{-/-}$mice compared with wild-type controls (Figure 3B). Intestinal granuloma formation was impaired both in size (Figure 3C) and number (Figure 3D) in the IL-21R $\mathrm{R}^{-/-}$mice following $\mathrm{Hp}$ infection. Splenocytes were isolated and restimulated for 3 days in the presence of $\mathrm{Hp}$ excretory secretory (HES) antigens, followed by a short restimulation with PMA and ionomycin. Surprisingly, intracellular cytokine staining and FACS analysis revealed that there was no significant difference in the proportion of IL-4- or IFN- $\gamma-$ producing $\mathrm{CD}^{+}{ }^{+} \mathrm{T}$ cells (Figure $3 \mathrm{E}$ ), although total cell counts were reduced overall (data not shown). These data indicated that the Th2 cells, which had survived the 3-day culture, were still capable of producing IL- 4 and IFN- $\gamma$, even in the absence of IL-21R signaling. In contrast, measurement of IL- 4 and IFN- $\gamma$ in the supernatant of the cultures by ELISA showed significantly reduced levels of IL-4 (Figure 3F) and increased levels of IFN- $\gamma$ (Figure $3 \mathrm{G}$ ) in the absence of IL-21R signaling. Taken together, these data indicate that while T cells can differentiate into Th2 cells in the absence of IL-21R signaling, they have impaired expansion or survival, and consequently in vivo and in vitro $\mathrm{Th} 2$ responses are impaired.

\section{IL-21R signaling is required for the development of Th2-driven allergic airway inflammation}

Systemic immunization followed by airway challenge with ovalbumin (OVA) is a standard method for inducing Th2-driven allergic airway inflammation, airways hyperresponsiveness, and recruit- ment of eosinophils. It has previously been reported that administration of exogenous IL-21 during immunization and challenge of mice with OVA impairs the ensuing recruitment of eosinophils into the airways. ${ }^{12}$ Suto et al ${ }^{12}$ showed that addition of IL-21 to T-helper cell cultures did not influence Th1 or Th2 differentiation, while other reports indicate that in vitro, IL-21 suppresses Th1 development and has no effect on Th2 cells. ${ }^{13}$ In an attempt to clarify such conflicting results, we immunized IL-21R ${ }^{-1-}$ and BALB/c control mice with OVA followed by intranasal challenge with OVA as described in "Materials and methods." In contrast to the report showing exogenous IL-21 can suppress eosinophil recruitment, ${ }^{12}$ the absence of IL-21R signaling in our system resulted in reduced eosinophil recruitment compared with BALB/c controls (Figure 4A). Lymphocyte migration was similarly impaired, indicating that IL-21 in fact supports lymphocyte expansion/migration and eosinophil maturation/migration to the airways. Expression of IL-21R on eosinophils was not detectable by RT-PCR, indicating a direct signal from IL-21 upon eosinophils was unlikely (data not shown). Ex vivo restimulation of OVA-specific lymphocytes led to reduced proliferation, suggesting expansion or survival of cells in vivo might also be impaired in the absence of IL-21R signaling (Figure 4B). In line with these data, IL-21 $\mathrm{R}^{-/-}$mice were less responsive to inhalation of increasing concentrations of MetCh, indicating that this IL-13-dependent airway hyperresponsiveness was also influenced by the absence of IL-21R signaling (Figure 4C). Notably, reduced levels of IL-13 in the BAL fluid of IL-21R ${ }^{-/-}$mice were also detected (data not shown). OVA-specific IgG1 (Figure 4D) and
A
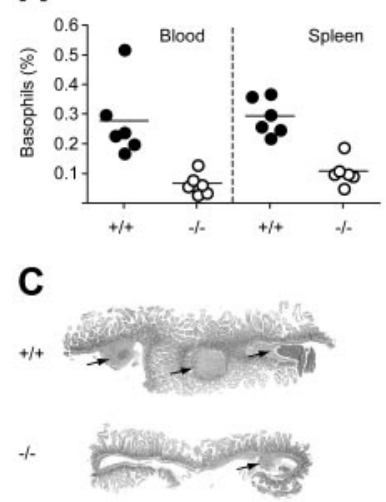

B

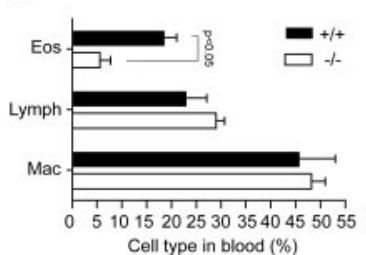

D

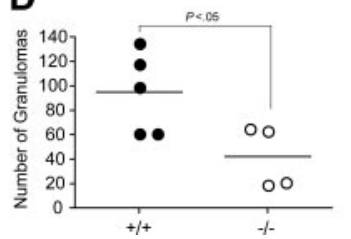

E
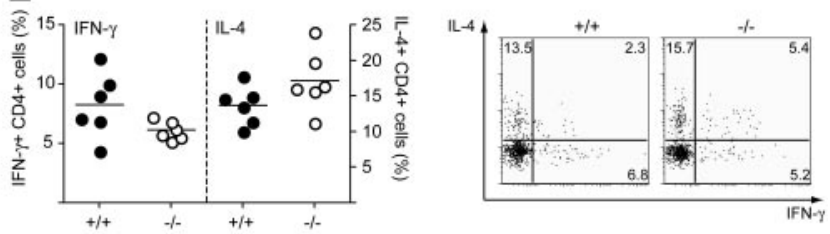

F

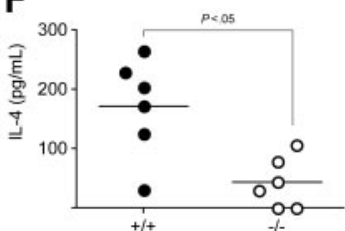

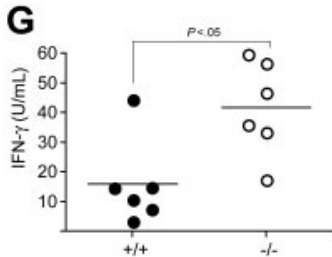

Figure 3. IL-21R signaling is required for Th2 responses against Heligmosomoides polygyrus. C57BL/6 and IL-21R-deficient mice were infected by oral gavage with $H$ polygyrus. On day 14 after infection, (A) the proportion of basophils in the blood and spleen were determined by FACS analysis. (B) The proportion of eosinophils circulating in the blood was determined by differential cell counts of blood smears. Data show averages \pm SD of a representative experiment using 5 to 6 mice per group. (C) H\&E staining of intestinal sections with granuloma indicated by arrows. (D) Granulomas were counted under a dissecting microscope. Splenocytes were cultured for 3 days in the presence of HES, followed by a short restimulation with PMA and ionomycin. (E) The proportion of CD4+ T cells producing IFN- $\gamma$ and IL-4 was determined by intracellular cytokine staining and FACS analysis. (F) IL-4 and (G) IFN- $\gamma$ from supernatant of the sample cultures were measured by ELISA. Similar results were obtained in a repeat experiment. (D-G) Circles indicate data of individual mice, and horizontal bars indicate averages of a group. 
Figure 4. IL-21R signaling is required for the development of Th2-driven allergic airway inflammation. BALB/C and IL-21R-deficient mice were immunized intraperitoneally with OVA protein adsorbed in alum adjuvant, followed 10 days later by intranasal challenge with OVA on 4 consecutive days. Two days after the final intranasal challenge (A) The number of eosinophils, lymphocytes, and macrophages that had infiltrated into the airways was determined by BAL and differential cell counts; (B) lung draining lymph nodes were removed and total lymph node cells cultured in the presence of OVA protein for 72 hours. $\left[{ }^{3} \mathrm{H}\right]$ thymidine incorporation over the last 12 hours of culture was measured as an indicator of cell proliferation. (C) One day after intranasal challenge, mice were exposed to increasing concentrations of acetyl- $\beta$ methacholine-chloride (MetCh) and airway hyperresponsiveness was determined using a full-body unrestrained plethysmograph. (D) OVA-specific $\lg G 1,(E) \lg A$, and $(F)$ total $\lg E$ were measured in BAL fluid. Data show averages $\pm S D$ of a representative experiment using 4 to 7 mice per group. Similar results were obtained in 3 independent experiments.
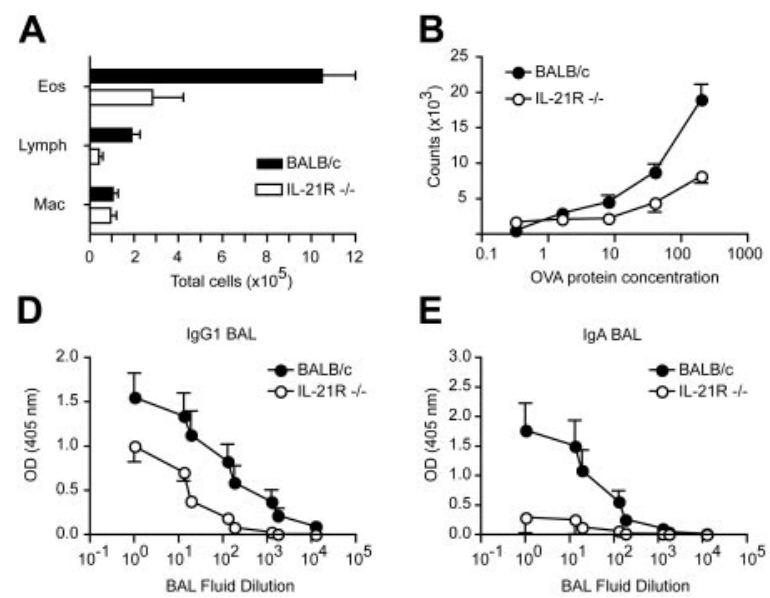
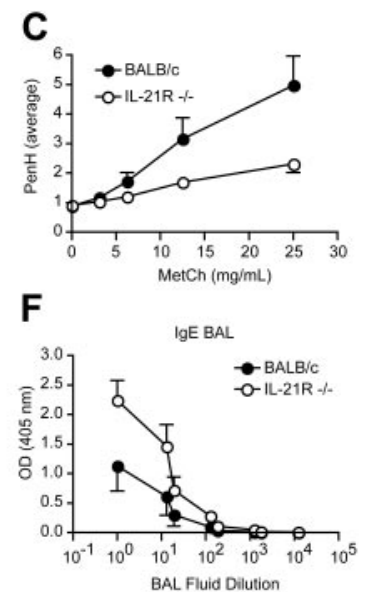

$\operatorname{Ig} \mathrm{A}$ (Figure 4E) in the BAL fluid and serum (data not shown) were reduced in the absence of the IL-21R; however, IgE production was enhanced (Figure $4 \mathrm{~F}$ ) as previously reported. ${ }^{10}$ Overall, these data indicate that IL-21 plays an important role in the development of Th2-mediated allergic airway inflammation.

\section{IL-21R $^{-1-}$ Th2 cells exhibit reduced recruitment or accumulation in the airways}

To assess whether the defect in Th2 responses was due to impaired T-cell priming by dendritic cells, we used an in vitro system where either C57BL/6 or IL-21R ${ }^{-1-}$ DCs were cultured together with TCR transgenic Sm2 CD4 $\mathrm{T}$ cells in the presence of $\mathrm{GP}_{61-80}$ peptide. After 24 hours, we analyzed the $\mathrm{T}$ cells for expression of activation markers (Figure 5A) and proliferation (Figure 5B). No differences between cells primed by C57BL/6 or IL-21R ${ }^{-1-}$ DCs were found, indicating IL-21R-signaling in DCs was not required for T-cell activation. We then analyzed the ability of CD4 T cells lacking the IL-21R to migrate to draining lymph nodes and

inflamed peripheral tissue in vivo. IL- $21^{+/+}$or $\mathrm{IL}-21^{-1-}$ TCR transgenic Sm2 CD4 cells, which can be tracked in vivo by expression of $\mathrm{V} \alpha 2$, were adoptively transferred into C57BL/6 mice. Recipient mice were immunized with $\mathrm{GP}_{61-80}$ peptide in alum adjuvant and after 10 days challenged with $\mathrm{GP}_{61-80}$ peptide intranasally. We analyzed draining LNs and the BAL for presence of transgenic CD4 $\mathrm{T}$ cells. While there was no difference in the percentage of $\mathrm{V} \alpha 2^{+} \mathrm{CD} 4^{+}$cells in the draining $\mathrm{LN}$ of either group (Figure 5C), we found less $\mathrm{Va} 2{ }^{+} \mathrm{CD}^{+}{ }^{+}$cells in the BALs of mice receiving IL-21 $\mathrm{R}^{-1-} \mathrm{Sm} 2 \mathrm{~T}$ cells (Figure $5 \mathrm{D}$ ). Total numbers of CD4 $\mathrm{T}$ cells in BALs were comparable in both groups, likely representing the nonspecific migration of endogenous memory $\mathrm{CD}^{+} \mathrm{T}$ cells to the site of inflammation. In addition, eosinophil numbers in the airways of mice receiving IL-21R $\mathrm{R}^{-1-} \mathrm{Sm} 2$ cells were reduced, supporting our previous findings with $\mathrm{Nb}$ infection and OVA-induced allergic airway inflammation (Figure 5E). Thus, while homing or survival of IL-21 $\mathrm{R}^{-1-} \mathrm{Sm} 2$ cells to the draining $\mathrm{LN}$ appears to be normal, the recruitment or survival of IL-21 $\mathrm{R}^{-/-}$

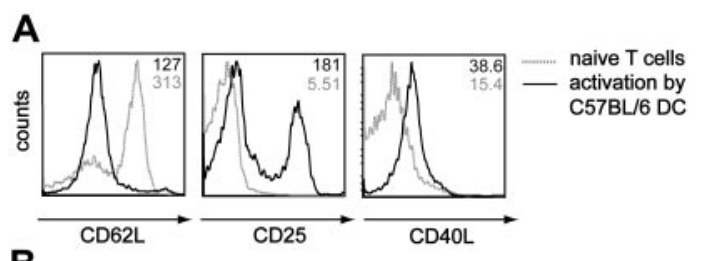

B
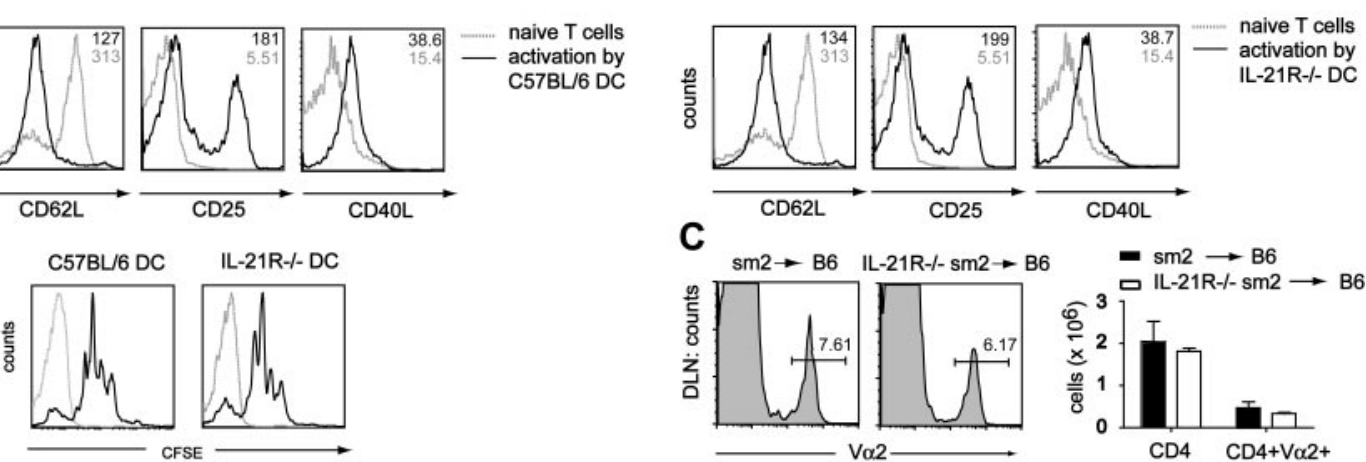

C
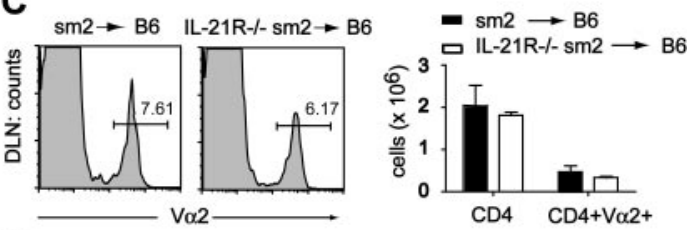

D

$\mathrm{sm} 2 \rightarrow \mathrm{B} 6 \quad \mathrm{IL}-21 \mathrm{R}-\mathrm{-}-\mathrm{sm} 2 \rightarrow \mathrm{B} 6$

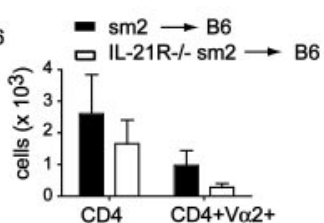

E
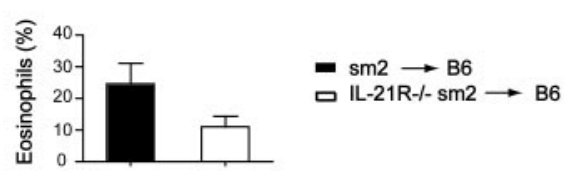

Figure 5. Normal T-cell priming but reduced T-cell recruitment to the airways in the absence of IL-21R signaling. DCs isolated from either C57BL/6 or IL-21R-deficient mice were cocultured with CFSE-labeled Sm2 T cells in the presence of $\mathrm{GP}_{61-80}$ peptide. (A) After 24 hours, cells were collected and stained for the activation markers CD62L, CD25, and CD40L. (B) Proliferation was assessed by FACS analysis of CFSE-stained cells. IL-21 ${ }^{+/+}$or IL-21 $1^{-/-}$TCR transgenic Sm2 CD4 cells were adoptively transferred into C57BL/6 mice. Mice were immunized with $\mathrm{GP}_{61-80}$ peptide in alum adjuvant. After 10 days, mice were challenged intranasally on 4 consecutive days. One day after the final challenge, the (C) DLNs and (D) BALs were analyzed for the percentage of the transferred Sm2 cells (Va2 ${ }^{+} \mathrm{CD} 4^{+}$) by FACS. (E) The number of eosinophils in the BAL was determined by differential cell counts. Numbers in histograms represent geometric mean; numbers above gates represent percentage of cells in gate. (C-E) Column plots indicate averages \pm SD of a representative experiment using 4 to 5 mice per group. Similar results were observed in 3 independent experiments. 


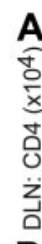
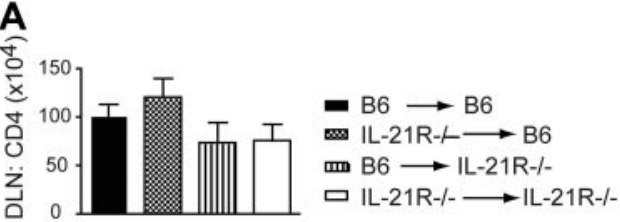

B
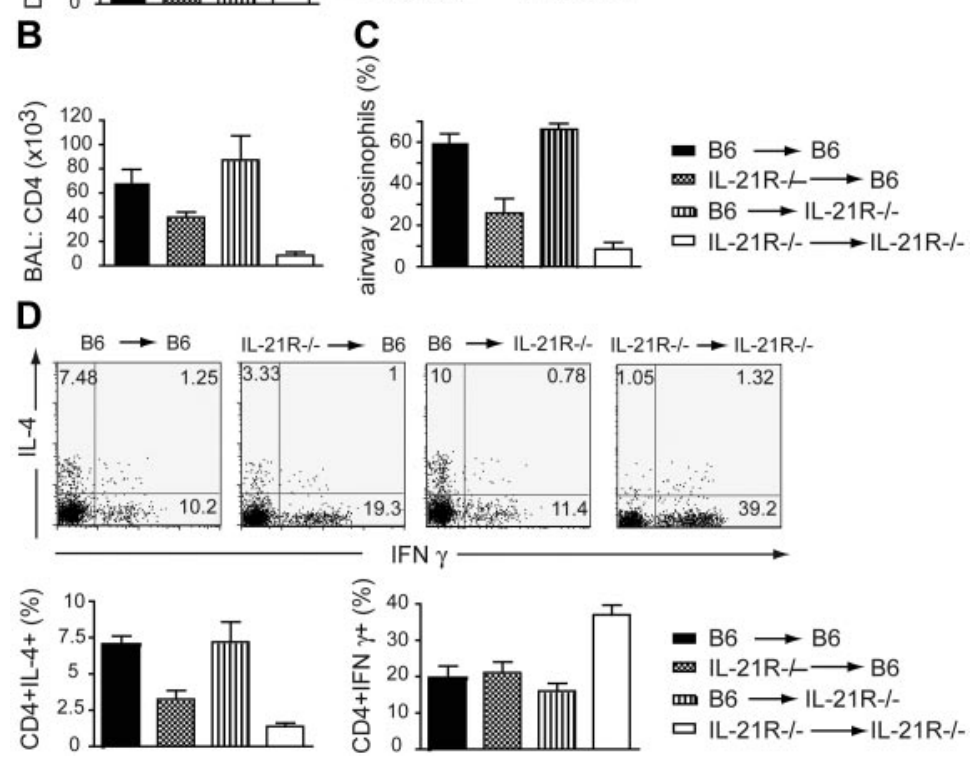

$\mathrm{CD}^{+} \mathrm{T}$ cells to the site of inflammation is impaired. As a consequence of the reduced number of Th2 cells in the airways, there is a reduced influx of eosinophils.

While it was likely that CD4 T cells were primarily accountable for the reduced Th2 responses, we also sought to clarify the contribution of nonhematopoietic cells. Accordingly, we lethally irradiated C57BL/6 mice or IL-21 $\mathrm{R}^{-1-}$ mice and reconstituted the hematopoietic compartment with bone marrow derived from either C57BL/6 or IL-21R ${ }^{-1-}$ mice. After successful reconstitution, we induced the model of allergic airway inflammation as described in "Materials and methods." Analysis of draining LNs showed no significant differences in the CD4 T-cell compartment (Figure 6A). In the BAL, however, a clear reduction of CD4 $\mathrm{T}$ cells in both groups that received IL-21 $\mathrm{R}^{-1-}$-derived $\mathrm{BM}$ compared with the groups that received $\mathrm{C} 57 \mathrm{BL} / 6 \mathrm{BM}$ was evident. In line with this, the number of eosinophils was strongly reduced in IL-21 ${ }^{-1-} \mathrm{BM}$ recipients (Figure 6B). IL-4 production by BAL-derived $\mathrm{CD}^{+}{ }^{+} \mathrm{T}$ cells was reduced in $\mathrm{IL}-21 \mathrm{R}^{-1-} \mathrm{BM}$ recipients, while IFN- $\gamma$ production was comparable or elevated (Figure 6C). These findings support the conclusion that the absence of IL-21R on CD4 T cells is the primary factor that leads to the overall reduced $\mathrm{Th} 2$ response. Notably, the impairment of $\mathrm{CD}^{+}{ }^{+} \mathrm{T}$ cell recruitment to the airways was slightly greater when IL- $21 \mathrm{R}^{-/-}$was absent in both the hematopoietic and nonhematopoietic compartments, indicating a secondary role for cells of the nonhematopoietic systems in the recruitment or accumulation of $\mathrm{CD} 4{ }^{+} \mathrm{T}$ cells to the airways.

\section{IL-21R signaling is not required to mount a resistant Th1 response against Leishmania major}

Th2 immune responses appear to be particularly sensitive to the absence of the IL-21R on T cells. In order to clarify the role of IL-21R signaling in Th1 immunity, we assessed the ability of IL-21R $\mathrm{R}^{-1-}$ mice on a C57BL/6 background to respond to $L$ major infection. Wild-type C57BL/6 mice respond to $L$ major
Figure 6. The absence of IL-21R on hematopoietic cells is primarily responsible for impaired Th2 immune responses in the lung. Reconstituted bone marrow chimeras were immunized intraperitoneally with OVA adsorbed in alum adjuvant, followed 10 days later by intranasal challenge with OVA on 4 consecutive days. Two days after the final challenge, the number of CD4 T cells in (A) DLN and (B) BAL was determined by FACS analysis. (C) Eosinophils that had infiltrated into the airways were determined by differential counts. (D) The proportion of $\mathrm{CD}^{+}{ }^{+} \mathrm{T}$ cells producing IL-4 and IFN- $\gamma$ was determined after PMA/ ionomycin restimulation by intracellular cytokine staining and FACS analysis. Column plots indicate averages $\pm \mathrm{SD}$ of a representative experiment using 4 mice per group. infection with a Th1 response dominated by the induction of IFN- $\gamma$ that enables macrophages to kill the parasite and clear the infection. ${ }^{16-18} \mathrm{IL}-21 \mathrm{R}^{-1-}$ and wild-type C57BL/6 mice were infected with $2 \times 10^{6}$ parasites in the right hind footpad. The inflammatory response was monitored weekly by measuring the swelling of the infected, compared with uninfected, footpad. No difference in footpad swelling was evident between IL-21 $\mathrm{R}^{-1-}$ and wild-type control mice (Figure 7A). Restimulation of lymphocytes isolated from the draining lymph node with $L$ major soluble antigens showed a significantly increased proportion of IFN- $\gamma$-producing $\mathrm{CD} 4^{+} \mathrm{T}$ cells from the IL-21R ${ }^{-1-}$ mice (Figure 7B), which became more pronounced at later time points (data not shown). These data indicate that IL-21R expression is not required for the development of Th1 immunity but, in fact, may suppress it.

\section{The development of Th17-driven experimental autoimmune myocarditis is independent of IL-21R signaling}

The role of Th1 and Th2 cytokines in the development of experimental autoimmune myocarditis (EAM) has been controversial. Recently, however, we found that EAM is in fact mediated by the Th17 cell subset. ${ }^{19}$ We sought to establish whether IL-21R signaling was involved in the development of Th17-mediated EAM. Mice were immunized with myosin peptide in CFA on days 0 and 7, and on day 21 were killed and hearts taken for histologic analysis. A comparable severity and prevalence of disease developed in both IL-21R $\mathrm{R}^{-/-}$and IL-21R $\mathrm{R}^{+/-}$littermate controls (Figure 7C). Histologic examination revealed the infiltrate in IL-21 $\mathrm{R}^{-/-}$ mice and IL-21 $1^{+/-}$littermate controls consisted primarily of macrophages and lymphocytes, with some granulocytes also being present (Figure 7D). No significant difference was detected in either the number or nature of the cell infiltrate. Overall, these data show that IL-21R signaling is superfluous for the development of Th17-mediated EAM. 
A

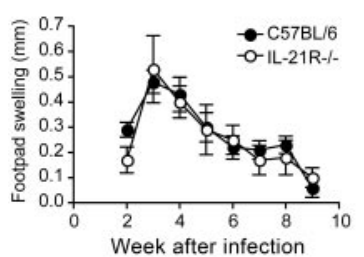

B

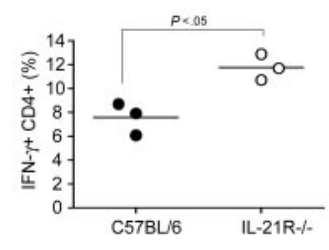

C

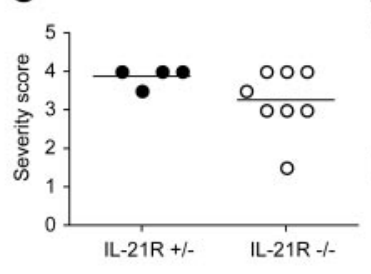

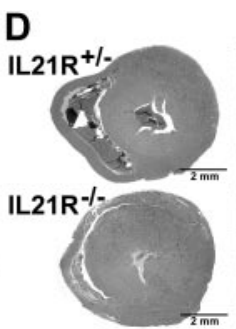

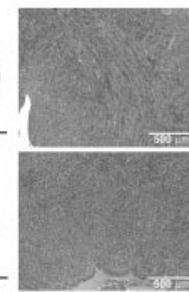

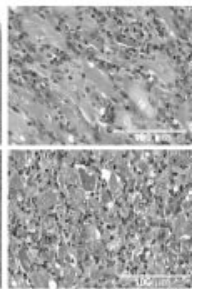

Figure 7. IL-21R-deficient mice exhibit a Th1 $\mathrm{R}^{-/-}$response following infection with Leishmania major and Th $17-\mathrm{mediated}$ protection from autoimmune myocarditis. (A,B) C57BL/6 and IL-21 (N5, C57BL/6) were infected with $2 \times 10^{6}$ promastigotes of $L$ major in the hind footpad. (A) At the time points indicated, the course of disease was monitored using metric calipers to determine footpad swelling. (B) Three weeks after infection, the draining lymph node was removed and isolated cells were restimulated with PMA and ionomycin for 4 hours. IFN- $\gamma$ production by $\mathrm{CD} 4^{+}$T cells was assessed by FACS. Values show percentage of IFN- $\gamma-$ producing cells, gated on $\mathrm{CD} 4{ }^{+}$ cells. Data are from a representative experiment using 3 to 5 mice per group. (C,D) IL-21R ${ }^{-/-}$(N4, BALB/c) and littermate control IL-21R ${ }^{+/-}$mice were immunized with $\alpha$-myosin-peptide in CFA on days 0 and 7 , and were killed 14 days later. Hearts were removed and fixed in $4 \%$ buffered formalin, and histological sections were evaluated. (C) The severity of inflammatory infiltrates were assessed and scored as described in "Materials and methods." Horizontal lines represent the median value for each group. (D) Representative images of H\&E-stained heart tissue. Original magnification, $15 \times$ (left column), $60 \times$ (middle column), and $400 \times$ (right column).

\section{Discussion}

Extensive research has shown that cytokines belonging to the type 1 cytokine family have a broad spectrum of activities, some of which might be considered conflicting. IL-4 for example is classically associated with the polarization and effector function of Th2 cells, ${ }^{20}$ and $\mathrm{IL}_{-} 4^{-1-}$ mice are defective in Th2 responses. ${ }^{21}$ However, it has also been reported that IL-4 promotes development of $\mathrm{CD}^{+}$cytotoxic $\mathrm{T}$ cells in tumor ${ }^{22}$ and viral ${ }^{23}$ models (typically associated with Th1, not Th2, responses). Similarly, IL-2 drives both Th1- and Th2-cell proliferation, while being important for stabilizing the accessibility of the Il4 gene and consequently Th2, not Th1, differentiation. ${ }^{24}$ IL-21 appears to follow suit in this respect, exhibiting pleiotropic effects in innate and adaptive immunity. A body of literature now supports a role for IL-21 in NK T-cell development and effector function, ${ }^{25-29}$ and B-cell antibody isotype switching. ${ }^{10,12,30-33}$ Furthermore, addition of IL-21 has been shown to drive IFN- $\gamma$ production in synergy with IL-15. ${ }^{28,34}$ However defining a role for IL-21 in Th1 and Th2 development remains controversial, possibly due to the nature of the in vitro systems used and the administration of exogenous cytokines.

In this paper, we have generated IL-21 receptor-deficient mice to allow the role of IL-21 during in vivo Th1, Th2, and Th17 disease models to be addressed. We first addressed Th2 immune responses, which are known to drive allergic airway inflammation and immunity against helminth parasites. In a model of OVA-induced allergic airway inflammation, IL-21R-deficient mice exhibited a reduced hyperresponsiveness upon inhalation of methacholine. In this model, AHR is typically associated with the activity of the Th2 cytokine IL-13, indicating that the IL-13 response was impaired in the absence of IL-21R signaling. In support of this result, analysis of the BAL fluid revealed reduced levels of IL-13 (data not shown). It has been shown that recruitment of eosinophils to the lung is dependent on the in vivo activity of IL-5, 35,36 but the question remained whether the reduced eosinophil recruitment was due to an inherent defect relating to the absence of the IL-21R, or whether it was a consequence of reduced lymphocyte migration into the airways and thus reduced release of IL-5. IL-21R expression was not detectible on eosinophils (data not shown), arguing against an inherent defect in these cells. Rather, the adoptive transfer experiments indicated that migration of IL-21R-deficient Sm2 T cells into the lung was impaired, while their survival and accumulation in the draining LN were comparable with wild-type T cells. These data were supported by the BM chimeras, which similarly showed reduced CD4 T-cell numbers in the BAL of IL-21Rdeficient BM recipients. Notably, the cytokine profiles of $\mathrm{IL}-21 \mathrm{R}^{-1-}$ $\mathrm{CD}^{+} \mathrm{T}$ cells that had reached the lung showed reduced $\mathrm{IL}-4$, and enhanced IFN- $\gamma$, production. The consequence of lower numbers of Th2 cells, and their reduced IL-4 production, is the likely mechanism underlying the reduced eosinophil recruitment and airway hyperresponsiveness. It remains to be definitively shown whether the reduced $\mathrm{CD}^{+} \mathrm{T}$-cell numbers in the lung are due to a reduced survival and accumulation or to an impairment in cell migration; however, given the nature of the common gamma chain family of cytokine receptors, impaired survival is the most plausible explanation.

In the absence of IL-21R signaling, T helper cell-dependent antigen-specific $\operatorname{IgA}$ and $\mathrm{IgG1}$ in the BAL were decreased in line with the reduced number of eosinophils and lymphocytes. While the impaired antibody response is likely to be secondary to the decreased $\mathrm{CD}^{+}{ }^{+}$T-cell response, it remains possible that the absence of the IL-21R on B cells may play a direct role.

Suto et $\mathrm{al}^{12}$ have shown that addition of IL-21 leads to reduced IgE and eosinophil migration in a model of OVA-induced allergic airway inflammation. While our data showing increased $\operatorname{IgE}$ production in the IL-21 $\mathrm{R}^{-1-}$ mice support their data, it is difficult to reconcile why eosinophil numbers and IgG1 production are reduced in both the absence of IL-21R and upon addition of IL-21. Further studies are required to clarify this issue, but possibilities include the following: IL-21 may play distinct roles at different stages of immune responses (potentially acting in synergy with different cytokines); its reported effect may be mediated through another cell type (eg, NK cells); or it might additionally signal through an as-yet-unidentified receptor.

An important question is whether IL-21, like its closest relative IL-2, is primarily involved in driving proliferation or polarization of T cells. The most well-accepted role for IL-2 is to enhance T-cell proliferation; however, it plays a clear role in Th2 differentiation. Our ex vivo proliferation experiments with OVA-specific T cells indicate that IL-21R signaling is critical for T-cell proliferation. Indeed, addition of IL-21 to in vitro cultures has been proved to enhance proliferation (Parrish-Novak et $\mathrm{al}^{6}$; Kasaian et $\mathrm{al}^{37}$; and data not shown), as does its closest related cytokine, IL-2. However IL-21 did not play a major role in mediating Th2-cell differentiation in vitro. ${ }^{13}$ The primary role of IL-21 in T-cell differentiation appears to support IL-4-mediated Th2 polarization, rather than to drive polarization itself. In line with this hypothesis, it has been shown that $\mathrm{T}$ cells isolated from IL-21R-deficient mice have no defect in polarization. ${ }^{10}$ However, exposure of naive $\mathrm{T}$ cells to IL-21 followed by culture in Th1-polarizing conditions resulted in a down-regulation of IFN- $\gamma$ expression, while IL-21 did not affect already polarized Th1 cells. ${ }^{13}$ We have also seen a reduction in IFN- $\gamma$ production upon addition of IL-21, which becomes more pronounced in the presence of IL-4 (A.F., B.J.M., M.K., 
unpublished data, March 2006). It is plausible that this phenomenon is linked to the well-established Th1/Th2 counterregulatory paradigm, with IL-21 playing a similar and supportive role to IL-4 in the suppression of Th1 development. However, considering IL-21 signals predominantly through STAT3 ${ }^{38}$ and IL-4 signals through STAT6, ${ }^{39}$ is it likely that the pathways underlying this may be distinct.

Given that a "resistant" phenotype against $L$ major is generally associated with effective Th1 immune responses, the comparable footpad swelling between C57BL/6 and IL-21R ${ }^{-1-}$ mice after infection with $L$ major suggests IL-21R signaling is not required for effective Th1mediated responses. Surprisingly, a significantly higher proportion of lymphocytes isolated from IL-21R ${ }^{-l-}$ mice, previously infected with $L$ major, produced IFN- $\gamma$, further supporting the hypothesis that IL-21 can impair Th1 polarization. Induction of experimental autoimmune myocarditis was also comparable between wild-type and IL-21R-deficient mice. These data suggest that any contribution of Th2 cytokines to the development of this disease must be minimal, or unnecessary, for normal disease development, and further indicate that IL-21 is not required for immune responses driven by IL-17. ${ }^{19}$

Clearly IL-21 exerts pleiotropic effects upon the development of innate and adaptive immune responses, and thus is a potential target for therapy. Our results would indicate that neutralizing IL-21 might be beneficial for the treatment of allergic asthma. However, while this could potentially be effective at reducing airway hyperresponsiveness and infiltration of lymphocytes, the resulting high circulating levels of IgE could have direct consequences. In addition, inhibiting the reported role for IL-21 in NK-cell effector function is unlikely to be therapeutically beneficial. Administering IL-21, however, might be a more promising alternative. Here, IgE production could be reduced, and potentially NK-cell function could be enhanced, although such treatment has been shown to exacerbate EAE. ${ }^{40}$ The implications these data have on potential therapeutics remain to be seen.

Overall, our data suggest the importance of IL-21R signaling in vivo may be on 3 distinct levels: (1) the suppression of IFN- $\gamma$ production; (2) the direct regulation of B-cell IgE isotype switching; and (3) enhancing T-cell survival. The pronounced sensitivity of Th2 responses to the absence of IL-21 signaling in vivo indicates that this cytokine is key to Th2, rather than Th1 or Th17, inflammation.

\section{Acknowledgments}

This work was supported by grants from the Swiss National Foundation (3100A0-100233/1) and ETH (TH 18/04-3).

\section{Authorship}

Contribution: A.F., I.S., and N.L.H. performed experiments; A.F., B.J.M., N.L.H., and M.K. designed and analyzed experiments; M.R.H. contributed vital new reagents; and A.F., B.J.M., and M.K. wrote the paper.

Conflict-of-interest disclosure: The authors declare no competing financial interests.

A.F. and B.J.M. contributed equally to this work.

Correspondence: Manfred Kopf, Molecular Biomedicine, ETH Zürich, Wagistrasse 27, 8952-Zürich-Schlieren, Switzerland; email: manfred.kopf@ethz.ch.

\section{References}

1. Kondo M, Takeshita T, Ishii N, et al. Sharing of the interleukin-2 (IL-2) receptor gamma chain between receptors for IL-2 and IL-4. Science. 1993; 262:1874-1877.

2. Sugamura K, Asao H, Kondo M, et al. The interleukin-2 receptor gamma chain: its role in the multiple cytokine receptor complexes and $\mathrm{T}$ cell development in XSCID. Annu Rev Immunol. 1996;14:179-205.

3. Di Santo JP, Kuhn R, Muller W. Common cytokine receptor gamma chain (gamma c)-dependent cytokines: understanding in vivo functions by gene targeting. Immunol Rev. 1995;148:19-34.

4. Noguchi M, Nakamura Y, Russell SM, et al. Interleukin-2 receptor gamma chain: a functional component of the interleukin-7 receptor. Science. 1993;262:1877-1880.

5. Russell SM, Keegan AD, Harada N, et al. Interleukin-2 receptor gamma chain: a functional component of the interleukin-4 receptor. Science. 1993;262:1880-1883.

6. Parrish-Novak J, Dillon SR, Nelson A et al. Interleukin 21 and its receptor are involved in NK cell expansion and regulation of lymphocyte function. Nature. 2000;408:57-63.

7. Ozaki K, Kikly K, Michalovich D, Young PR, Leonard WJ. Cloning of a type I cytokine receptor most related to the IL-2 receptor beta chain. Proc Natl Acad Sci U S A. 2000;97:11439-11444.

8. Ma HL, Whitters MJ, Konz RF, et al. IL-21 activates both innate and adaptive immunity to generate potent antitumor responses that require perforin but are independent of IFN-gamma. J Immunol. 2003;171:608-615.

9. Brandt K, Bulfone-Paus S, Jenckel A, Foster DC, Paus R, Ruckert R. Interleukin-21 inhibits dendritic cell-mediated $T$ cell activation and induction of contact hypersensitivity in vivo. J Invest Dermatol. 2003:121:1379-1382.
10. Ozaki K, Spolski R, Feng CG, et al. A critical role for IL-21 in regulating immunoglobulin production. Science. 2002;298:1630-1634.

11. Leonard WJ, Spolski R. Interleukin-21: a modula tor of lymphoid proliferation, apoptosis and differentiation. Nat Rev Immunol. 2005;5:688-698.

12. Suto A, Nakajima H, Hirose $K$, et al. Interleukin 21 prevents antigen-induced IgE production by inhibiting germ line $\mathrm{C}$ (epsilon) transcription of IL-4stimulated B cells. Blood. 2002;100:4565-4573.

13. Wurster AL, Rodgers VL, Satoskar AR, et al. In terleukin 21 is a Thelper (Th) cell 2 cytokine that specifically inhibits the differentiation of naive Th cells into interferon gamma-producing Th1 cells. J Exp Med. 2002;196:969-977.

14. Strengell M, Sareneva T, Foster D, Julkunen I, Matikainen S. IL-21 up-regulates the expression of genes associated with innate immunity and Th1 response. J Immunol. 2002;169:3600-3605.

15. Ruedl C, Bachmann MF, Kopf M. The antigen dose determines $T$ helper subset development by regulation of CD40 ligand. Eur J Immunol. 2000; 30:2056-2064

16. Heinzel FP, Sadick MD, Holaday BJ, Coffman RL Locksley RM. Reciprocal expression of interferon gamma or interleukin 4 during the resolution or progression of murine leishmaniasis: evidence for expansion of distinct helper T cell subsets. J Exp Med. 1989;169:59-72.

17. Reiner SL, Locksley RM. The regulation of immunity to Leishmania major. Annu Rev Immunol. 1995;13:151-177.

18. Sacks D, Noben-Trauth $N$. The immunology of susceptibility and resistance to Leishmania major in mice. Nat Rev Immunol. 2002;2:845-858.

19. Sonderegger I, Till R, Kurrer MO, et al. Neutralization of IL- 17 by active vaccination inhibits IL-23 mediated experimental autoimmune myocarditis. Eur J Immunol. 2006;36:1-8.
20. Le Gros G, Ben-Sasson SZ, Seder R, Finkelman FD, Paul WE. Generation of interleukin 4 (IL-4)producing cells in vivo and in vitro: IL-2 and IL-4 are required for in vitro generation of IL-4-producing cells. J Exp Med. 1990;172:921-929.

21. Kopf M, Baumann H, Freer G, et al. Impaired immune and acute-phase responses in interleukin6-deficient mice. Nature. 1994;368:339-342.

22. Schuler T, Kammertoens T, Preiss S, Debs P, Noben-Trauth N, Blankenstein T. Generation of tumor-associated cytotoxic $T$ lymphocytes requires interleukin 4 from CD8(+) T cells. J Exp Med. 2001;194:1767-1775.

23. Marsland BJ, Schmitz N, Kopf M. IL-4Ralpha signaling is important for CD8 + T cell cytotoxicity in the absence of CD4+ T cell help. Eur J Immunol. 2005;35:1391-1398.

24. Cote-Sierra J, Foucras G, Guo L, et al. Interleukin 2 plays a central role in Th2 differentiation. Proc Natl Acad Sci U S A. 2004;101:3880-3885.

25. Takaki R, Hayakawa Y, Nelson A, et al. IL-21 enhances tumor rejection through a NKG2Ddependent mechanism. J Immunol. 2005;175:21672173.

26. Brady J, Hayakawa Y, Smyth MJ, Nutt SL. IL-21 induces the functional maturation of murine NK cells. J Immunol. 2004;172:2048-2058.

27. Sivori S, Cantoni C, Parolini S, et al. IL-21 induces both rapid maturation of human CD34+ cell precursors towards NK cells and acquisition of surface killer Ig-like receptors. Eur J Immunol. 2003;33:3439-3447.

28. Strengell M, Matikainen S, Siren J, et al. IL-21 in synergy with IL-15 or IL-18 enhances IFN-gamma production in human NK and T cells. J Immunol. 2003;170:5464-5469.

29. Wang G, Tschoi M, Spolski R, et al. In vivo antitumor activity of interleukin 21 mediated by natural killer cells. Cancer Res. 2003;63:9016-9022. 
From www.bloodjournal.org at ECOLE POLYTECHNIQUE FEDERALE DE LAUSANNE on January 6, 2010. For BLOOD, 1 MARCH 2007 • VOLUME 109, NUMBER 5

30. Ettinger R, Sims GP, Fairhurst AM, et al. IL-21 induces differentiation of human naive and memory B cells into antibody-secreting plasma cells. J Immunol. 2005;175:7867-7879.

31. Wood N, Bourque K, Donaldson DD, et al. IL-21 effects on human IgE production in response to IL-4 or IL-13. Cell Immunol. 2004;231:133-145.

32. Ozaki K, Spolski R, Ettinger R, et al. Regulation of $B$ cell differentiation and plasma cell generation by IL-21, a novel inducer of Blimp-1 and Bcl-6. J Immunol. 2004;173:5361-5371.

33. Pene J, Gauchat JF, Lecart S, et al. Cutting edge: $\mathrm{IL}-21$ is a switch factor for the production of $\operatorname{lgG} 1$ and IgG3 by human B cells. J Immunol. 2004; 172:5154-5157.

34. Zeng R, Spolski R, Finkelstein SE, et al. Synergy of $\mathrm{IL}-21$ and IL-15 in regulating CD8 + T cell expansion and function. J Exp Med. 2005;201:139-148.

35. Dent LA, Strath M, Mellor AL, Sanderson CJ. Eosinophilia in transgenic mice expressing interleukin 5. J Exp Med. 1990;172:1425-1431.

36. Yamaguchi Y, Suda T, Suda J, et al. Purified interleukin 5 supports the terminal differentiation and proliferation of murine eosinophilic precursors. J Exp Med. 1988;167:43-56.

37. Kasaian MT, Whitters MJ, Carter LL, et al. IL-21 limits NK cell responses and promotes antigen- specific T cell activation: a mediator of the transition from innate to adaptive immunity. Immunity. 2002;16:559-569.

38. Asao H, Okuyama C, Kumaki S, et al. Cutting edge: the common gamma-chain is an indispensable subunit of the IL-21 receptor complex. J Immunol. 2001;167:1-5.

39. Hou J, Schindler U, Henzel WJ, Ho TC, Brasseur M, McKnight SL. An interleukin-4-induced transcription factor: IL-4 Stat. Science. 1994;265:1701-1706.

40. Vollmer TL, Liu R, Price M, Rhodes S, La Cava A, Shi FD. Differential effects of IL-21 during initiation and progression of autoimmunity against neuroantigen. J Immunol. 2005;174:2696-2701. 